\title{
Anesthetic requirements and stress hormone responses in chronic spinal cord-injured patients undergoing surgery below the level of injury: nitrous oxide vs remifentanil
}

\author{
Dong Ho Kang ${ }^{1}$, Seong-Heon Lee ${ }^{1}$, Seok Jai Kim ${ }^{1}$, Jeong-Il Choi ${ }^{1}$, Cheol-Won Jeong ${ }^{2}$, Seong Wook \\ Jeong $^{1}$, and Kyung Yeon Yoo ${ }^{1}$ \\ Department of Anesthesiology and Pain Medicine, ${ }^{1}$ Chonnam Natioal University Medical School, ${ }^{2}$ School of Dentistry, Chonnam \\ National University, Gwangju, Korea
}

Background: Nitrous oxide $\left(\mathrm{N}_{2} \mathrm{O}\right)$ and remifentanil both have anesthetic-reducing and antinociceptive effects. We aimed to determine the anesthetic requirements and stress hormone responses in spinal cord-injured (SCI) patients undergoing surgery under sevoflurane anesthesia with or without pharmacodynamically equivalent doses of $\mathrm{N}_{2} \mathrm{O}$ or remifentanil.

Methods: Forty-five chronic, complete SCI patients undergoing surgery below the level of injury were randomly allocated to receive sevoflurane alone (control, $n=15)$, or in combination with $67 \% \mathrm{~N}_{2} \mathrm{O}(\mathrm{n}=15)$ or target-controlled infusion of $1.37 \mathrm{ng} / \mathrm{ml}$ remifentanil $(\mathrm{n}=15)$. Sevoflurane concentrations were titrated to maintain a Bispectral Index (BIS) value between 40 and 50. Measurements included end-tidal sevoflurane concentrations, mean arterial blood pressure (MAP), heart rate (HR), and plasma catecholamine and cortisol concentrations.

Results: During surgery, MAP, HR, and BIS did not differ among the groups. Sevoflurane concentrations were lower in the $\mathrm{N}_{2} \mathrm{O}$ group $(0.94 \pm 0.30 \%)$ and the remifentanil group $(1.06 \pm 0.29 \%)$ than in the control group $(1.55 \pm 0.34 \%)$ $(\mathrm{P}<0.001$, both). Plasma concentrations of norepinephrine remained unchanged compared to baseline values in each group, with no significant differences among groups throughout the study. Cortisol levels decreased during surgery as compared to baseline values, and returned to levels higher than baseline at $1 \mathrm{~h}$ after surgery $(\mathrm{P}<0.05)$ without intergroup differences.

Conclusions: Remifentanil ( $1.37 \mathrm{ng} / \mathrm{ml})$ and $\mathrm{N}_{2} \mathrm{O}(67 \%)$ reduced the sevoflurane requirements similarly by $31-39 \%$, with no significant differences in hemodynamic and neuroendocrine responses. Either remifentanil or $\mathrm{N}_{2} \mathrm{O}$ can be used as an anesthetic adjuvant during sevoflurane anesthesia in SCI patients undergoing surgery below the level of injury. (Korean J Anesthesiol 2013; 65: 531-538)

Key Words: Catecholamines, Nitrous oxide, Remifentanil, Sevoflurane, Spinal cord injuries.

Received: May 28, 2013. Accepted: June 20, 2013.

Corresponding author: Kyung Yeon Yoo, M.D., Ph.D., Department of Anesthesiology and Pain Medicine, Chonnam National University Hospital, 42, Jebong-ro, Jung-gu, Gwangju 501-757, Korea. Tel: 82-62-220-6893, Fax: 82-62-232-6294, E-mail: kyyoo@jnu.ac.kr

(c) This is an open-access article distributed under the terms of the Creative Commons Attribution Non-Commercial License (http:// creativecommons.org/licenses/by-nc/3.0/), which permits unrestricted non-commercial use, distribution, and reproduction in any medium, provided the original work is properly cited. 


\section{Introduction}

Spinal or epidural anesthesia is associated with an increased depth of hypnosis and anesthesia [1,2], presumably via an interruption of the ascending somatosensory pathway to the brain. A complete spinal cord injury (SCI) results in sensory and motor deficits as well as an interruption of sympathetic outflow below the injury level [3]. It may simulate spinal or epidural anesthesia in terms of spinal deafferentation. Moreover, SCI produces abrupt irreversible deafferentation of cortical circuits and changes the state of large cortical networks, leading to cortical reorganization [4]. In this context, SCI may alter anesthetic requirements as well as cardiovascular and stress responses to surgical stimuli by an anesthetic-sparing effect or interruption of sympathetic efferent fibers innervating the cardiovascular system including the heart.

Patients with SCI have a reduced sympathetic activity, and are highly susceptible to hypotension under general anesthesia [5]. Many adjuvant drugs when combined with volatile anesthetics have anesthetic-sparing properties. Among them, nitrous oxide $\left(\mathrm{N}_{2} \mathrm{O}\right)$ has been used during general anesthesia because of its many desirable properties, including minimum alveolar concentration (MAC)-reducing effects, low solubility with a rapid onset and offset of action, and limited cardiovascular and respiratory depression [6,7]. However, there are concerns regarding its adverse effects, such as risk of hypoxia, expansion of air-filled spaces, myocardial ischemia, postoperative nausea and vomiting, and megaloblastic anemia [6]. Remifentanil is an ultra short-acting opioid, providing a potent analgesia of rapid onset [8]. It has been demonstrated that remifentanil significantly reduces the volatile anesthetic concentration required to abolish consciousness, and blunt the autonomic response to surgical stimuli [9]. However, the use of remifentanil is associated with hypotension and bradycardia [8].

In earlier studies by our group, the amount of sevoflurane required to maintain BIS at 40-50 in SCI patients undergoing surgery below the level of injury was reduced by $20-39 \%$ in the presence of $50 \% \mathrm{~N}_{2} \mathrm{O}$ compared with that in normal healthy controls $[10,11]$. However, the interaction between $\mathrm{N}_{2} \mathrm{O}$ or remifentanil and volatile anesthetics at the cortical level is unclear in these patients in whom sensory afferences to the brain are lacking. Anesthetics act in the spinal cord to ablate movement in response to a noxious stimulus, and in the brain to cause amnesia and unconsciousness [12]. Their action in the brain can influence the spinal cord; however, the extent probably varies among anesthetics [13]. Conversely, the spinal action can indirectly influence the brain, and hence the level of amnesia and unconsciousness [13]. $\mathrm{N}_{2} \mathrm{O}$ has been shown to have multiple sites of action in the spinal cord and brain [14]. Remifentanil exerts its effects on the $\mu$-opioid receptors, primarily those located in the dorsal horn of the spinal cord, periaqueductal gray, and locus coeruleus. Therefore, the current study was aimed to determine the interaction between antinociceptive agents, namely $\mathrm{N}_{2} \mathrm{O}$ or remifentanil and sevoflurane at the cortical level, and/or the neuroendocrine response in SCI patients undergoing surgery below the level of injury.

\section{Materials and Methods}

After the University Hospital Ethics Committee approval, written informed consent was obtained from all patients. Fortyfive patients with chronic, clinically complete spinal cord injuries scheduled for surgery for pressure ulcer below the level of injury under general anesthesia were randomly assigned to receive either sevoflurane alone (control, $\mathrm{n}=15$ ) or in combination with $\mathrm{N}_{2} \mathrm{O}(\mathrm{n}=15)$ or remifentanil $(\mathrm{n}=15)$, based on a computergenerated randomization list. Patients in whom the time lag between injury and surgery was less than 6 weeks were excluded, as it takes up to 4-6 weeks to recover muscle tone and reflexes in the spinal cord distal to the site of injury [4]. Patients who took medications that would influence the autonomic or cardiovascular response to surgical stress were also excluded. Neurologic examinations of all disabled patients were performed by our University Hospital Spine Center according to 1996 American Spinal Injury Association standards.

All patients were premedicated with midazolam $0.1 \mathrm{mg} / \mathrm{kg}$ orally $60 \mathrm{~min}$ before being transported to the operating room. Before anesthesia, a venous catheter was inserted and infusion of lactated Ringer's solution was initiated at a rate of $10 \mathrm{ml} / \mathrm{kg} /$ h. In addition, a 20-gauge catheter was inserted into a radial artery to measure blood pressure and take blood samples. A BIS sensor electrode (BIS ${ }^{\circledR}$ Sensor-Aspect Medical Systems, Natick, MA, USA) was placed on the forehead as recommended by the manufacturer.

After full preoxygenation, anesthesia was induced with intravenous (IV) sodium thiopental $5 \mathrm{mg} / \mathrm{kg}$ and remifentanil $1 \mu \mathrm{g} /$ $\mathrm{kg}$, followed by rocuronium $0.8 \mathrm{mg} / \mathrm{kg}$. The trachea was intubated when neuromuscular block was achieved; and anesthesia was maintained with either sevoflurane alone (control), or in combination with $67 \% \mathrm{~N}_{2} \mathrm{O}$ or target-controlled infusion (TCI) of 1.37 $\mathrm{ng} / \mathrm{ml}$ remifentanil according to the group to which the patient was assigned. This concentration of remifentanil was selected because it reduced the MAC of isoflurane by $50 \%$ [9], which is similar to the degree of MAC reduction expected with the use of $67 \% \mathrm{~N}_{2} \mathrm{O}$ [7]. Remifentanil was administered using the pharmacokinetic model described by Minto et al. via an effect-site TCI device (Orchestra ${ }^{\circledR}$ Base Primea, Fresenius Kabi, Brezins, Germany). In clinical practice, when using conventional weightadjusted dose administration, the effect-site TCI concentration of remifentanil of $1.37 \mathrm{ng} / \mathrm{ml}$ used in this study can be achieved 
by administering a $1 \mu \mathrm{g} / \mathrm{kg}$ bolus over $60 \mathrm{~s}$ followed by a infusion of $0.05 \mu \mathrm{g} / \mathrm{kg} / \mathrm{min}$. Patients' lungs were ventilated using positive pressure ventilation to maintain end-tidal carbon dioxide tension between 35 and $40 \mathrm{mmHg}$ with $\mathrm{N}_{2} \mathrm{O}$ or air in oxygen (inspired fraction of 0.33 ). Since BIS could be highly contaminated by electromyographic activity, additional boluses of rocuronium (10 mg) were administered to maintain full muscle relaxation (no twitch response to train-of-four stimulation) during surgery. Routine monitoring including invasive measurement of systemic blood pressure, heart rate (HR) and rhythm by ECG and pulse oximetry were performed. Throughout the study, the endtidal concentrations of carbon dioxide, sevoflurane, and $\mathrm{N}_{2} \mathrm{O}$ were measured using a gas analyzer (Capnomac Ultima; DatexOhmeda, Helsinki, Finland).

The concentration of sevoflurane was adjusted to maintain the BIS value between 40 and 50 [15]. Bolus doses of fentanyl $1 \mu \mathrm{g} / \mathrm{kg}$ were given if the mean arterial blood pressure (MAP) and/or HR increased by $>20 \%$ above baseline for more than 1 min or if patients tear, sweat or move in response to a surgical stimulus. If the response was present 2 min later, fentanyl was administered repeatedly. Hypotension, defined as a decrease in MAP more than 30\% from the baseline value for more than 1 min, which did not respond to a fluid bolus, was treated with IV boluses of ephedrine $8 \mathrm{mg}$.

At the end of surgery, volatile anesthetics were discontinued and residual neuromuscular block was reversed with pyridostigmine and glycopyrrolate when 2 or more twitch responses to train-of-four stimulation were observed. Estimated blood loss and amount of fluid or blood administered during surgery were recorded. Postoperative pain intensity was estimated using a 10 $\mathrm{cm}$ visual analog scale (VAS). Pain was treated using IV fentanyl and propacetamol to achieve a VAS score $<4$. The day after surgery, all of the studied patients were interviewed to evaluate the presence of explicit recall of any intraoperative event.

MAP and HR were recorded before anesthesia induction (awake), immediately before the beginning of surgery, every 5 min for $1 \mathrm{~h}$ during surgery and at $1 \mathrm{~h}$ after surgery. The values recorded during the first hour of surgery were averaged to provide a single mean value. End-tidal concentrations of sevoflurane and $\mathrm{N}_{2} \mathrm{O}$, and $\mathrm{BIS}$ values were similarly averaged. We analyzed the hemodynamic and stress hormonal data for the first one hour of surgery only because of a different length of surgery in each patient.

Arterial blood samples for measurement of catecholamine and cortisol concentrations were obtained before anesthesia induction (baseline), immediately before the beginning of surgery, at $1 \mathrm{~h}$ of surgery, and $1 \mathrm{~h}$ after the end of surgery. The samples were collected into pre-chilled tubes containing EDTA-Na and immediately centrifuged at $3000 \mathrm{rpm}$ for $10 \mathrm{~min}$ at $4^{\circ} \mathrm{C}$. The plasma was stored at $-70^{\circ} \mathrm{C}$ until assayed. Plasma concentra- tions of norepinephrine and epinephrine were measured in duplicate by using high performance liquid chromatography [10]. The assay sensitivity was $25 \mathrm{pg} / \mathrm{ml}$ and $10 \mathrm{pg} / \mathrm{ml}$ and within-run precision coefficients of variation were $4.2 \%$ and $7.8 \%$ for norepinephrine and epinephrine, respectively. Plasma concentrations of cortisol were determined by radioimmunoassay (DSL2000 SP Aktive ${ }^{\circledR}$ Cortisol; Diagnostic System Laboratories, Sinsheim, Germany).

Sample size was calculated to detect a 30\% difference in sevoflurane requirement between the $\mathrm{N}_{2} \mathrm{O}$ group and the remifentanil group, based on the preliminary study ( $\mathrm{n}=5$ each). Thirteen patients were required in each group to detect an effect size of 1.17 in an analysis using an independent t-test with an $\alpha$ of 0.05 and a power of 0.80 . Fifteen patients were enrolled in each group to compensate for possible dropouts. Statistical analysis was performed using the program StatView 4.0 (Abacus Concepts, Berkeley, CA, USA). Sex data were analyzed using the Fisher's exact test. Other demographic and surgical data were compared using one-way analysis of variance. Differences between the groups of variables (MAP, HR, BIS values, sevoflurane concentrations, and stress hormones) were analyzed by two-way repeated measures analysis of variance. A Scheffe' test was used for multiple pairwise comparisons when a significant difference was indicated by the analysis of variance. Normal distribution of continuous variables was determined using the KolmogorovSmirnov test (all $P$ values $>0.05$ ). Data are presented as mean \pm SD. A P value of 0.05 or less was considered statistically significant.

\section{Results}

Among 53 subjects initially enrolled in the study, 8 were excluded; 4 did not meet the inclusion criteria, 3 declined to participate in the study, and 1 was taking $\beta$-blockers before surgery. The resultant 45 subjects were randomized in equal numbers to the three groups, and they completed the study without any protocol violations (Fig. 1). Demographic and surgical data were comparable among the three groups (Table 1). Total operative time, intraoperative fluid requirement, and blood loss did not significantly differ between groups. Of the 45 studied patients, the level of injury was between C5 and T4 in 14 patients and between T5 and L3 in 31 patients (Fig. 2). None of the patients in any group required analgesics during recovery. The day after the surgery, none of the patients recalled awareness during anesthesia and surgery.

Anesthetic induction decreased the MAP and BIS values ( $P$ $<0.0001$ ), but it did not affect the HR. The MAP, HR and BIS values did not differ significantly between before surgery (immediately before beginning the surgery) and during surgery in any group (Table 2). Before surgery and during surgery, end- 
tidal sevoflurane concentrations were significantly lower in the $\mathrm{N}_{2} \mathrm{O}$ group (0.94-0.98\%) and the remifentanil group (1.06$1.09 \%)$ than in the control group $(1.55-1.57 \%)(\mathrm{P}<0.001$, both); however, there were no differences between the two experimental groups (Fig. 3). None of the patients in any group received fentanyl, whereas 1 patient in the remifentanil group received ephedrine ( $8 \mathrm{mg}$ ) during surgery. One hour after the surgery, the MAP and HR did not differ significantly from their baseline values in each group nor did they differ significantly between groups at any time throughout the study.

Table 3 shows the plasma concentrations of catecholamines and cortisol. Their preoperative baseline values did not differ significantly among groups. Plasma norepinephrine levels remained unaltered throughout the study. Epinephrine levels

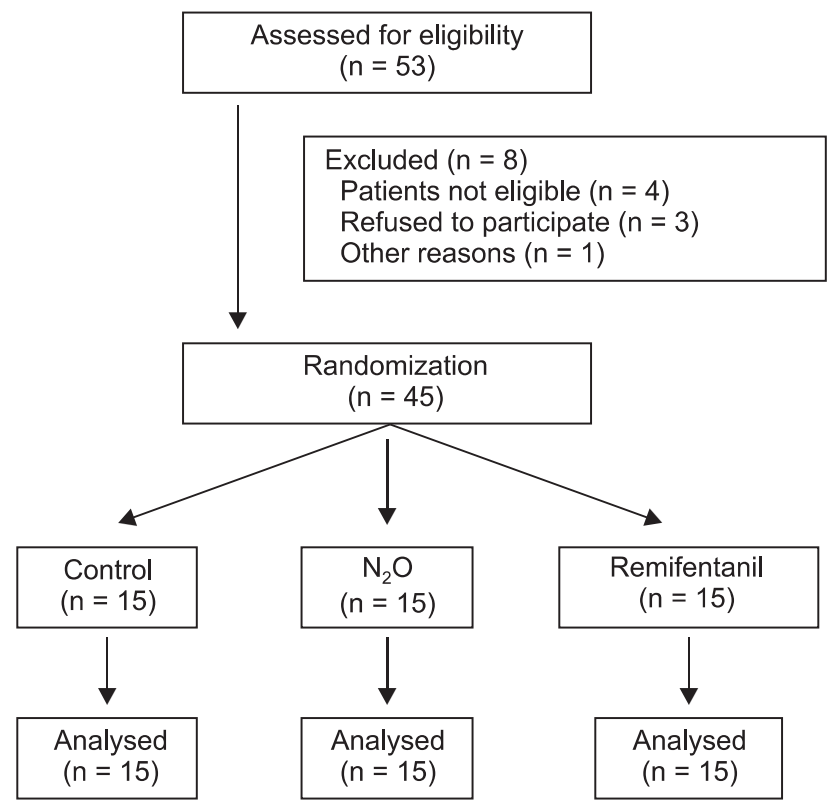

Fig. 1. CONSORT flow chart showing the flow of subjects through the trial. decreased after induction of anesthesia (i.e., before surgery) in all groups $(\mathrm{P}<0.05)$, which recovered to the baseline values at 1 hour after the surgery. However, cortisol levels were significantly decreased during surgery as compared to baseline values and rose above baseline values at $1 \mathrm{~h}$ after the end of surgery

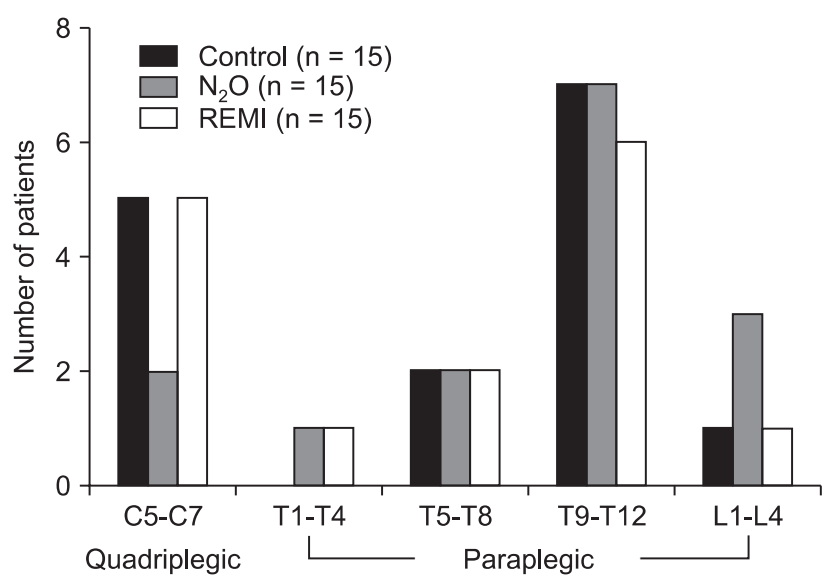

Fig. 2. Distribution of injury levels in patients given sevoflurane alone or in combination with nitrous oxide $\left(\mathrm{N}_{2} \mathrm{O}\right)$ or remifentanil (REMI).

Table 1. Demographic and Surgical Data in Patients Given Sevoflurane Alone (Control) or in Combination with Nitrous Oxide $\left(\mathrm{N}_{2} \mathrm{O}\right)$ or Remifentanil (REMI)

\begin{tabular}{lccc}
\hline \multicolumn{1}{c}{ Variable } & $\begin{array}{c}\text { Control } \\
(\mathrm{n}=15)\end{array}$ & $\begin{array}{c}\mathrm{N}_{2} \mathrm{O} \\
(\mathrm{n}=15)\end{array}$ & $\begin{array}{c}\text { REMI } \\
(\mathrm{n}=15)\end{array}$ \\
\hline Sex (M/F) & $14 / 1$ & $14 / 1$ & $13 / 2$ \\
Age (yr) & $48 \pm 15$ & $41 \pm 11$ & $47 \pm 14$ \\
Height (cm) & $171 \pm 7$ & $171 \pm 6$ & $169 \pm 6$ \\
Body weight (kg) & $62 \pm 12$ & $62 \pm 13$ & $59 \pm 10$ \\
Hemoglobin (g/dl) & $12.2 \pm 1.3$ & $12.9 \pm 1.4$ & $11.9 \pm 1.2$ \\
Duration of surgery (min) & $160 \pm 52$ & $159 \pm 55$ & $152 \pm 76$ \\
Fluid requirement (ml) & $1830 \pm 935$ & $1997 \pm 669$ & $1993 \pm 1029$ \\
Post injury (months) & $132 \pm 135$ & $152 \pm 110$ & $117 \pm 122$ \\
\hline
\end{tabular}

Values are mean \pm SD or absolute numbers. No significant differences were noted among the groups.

Table 2. Mean Arterial Blood Pressure (MAP), Heart rate (HR), and Bispectral Index Values (BIS) before Induction of Anesthesia, before, During and 1 Hour after (Recovery) Surgery in Patients Given Sevoflurane Alone (Control, $n=15)$ or in Combination with Nitrous Oxide $\left(\mathrm{N}_{2} \mathrm{O}, \mathrm{n}=15\right)$ or Remifentanil (REMI, $\mathrm{n}=15)$

\begin{tabular}{clcccc}
\hline \multicolumn{1}{c}{ Variable } & Group & Before induction & Before surgery & During surgery & Recovery \\
\hline MAP (mmHg) & Control & $88 \pm 12$ & $74 \pm 7^{*}$ & $77 \pm 11^{*}$ & $90 \pm 13$ \\
& $\mathrm{~N}_{2} \mathrm{O}$ & $96 \pm 14$ & $82 \pm 13^{*}$ & $80 \pm 12^{*}$ & $96 \pm 10$ \\
& $\mathrm{REMI}$ & $98 \pm 16$ & $77 \pm 10^{*}$ & $80 \pm 9^{*}$ & $91 \pm 17$ \\
$\mathrm{HR}(\mathrm{bpm})$ & $\mathrm{Control}$ & $74 \pm 8$ & $75 \pm 14$ & $70 \pm 14$ & $73 \pm 14$ \\
& $\mathrm{~N}_{2} \mathrm{O}$ & $78 \pm 10$ & $80 \pm 9$ & $74 \pm 13$ & $76 \pm 8$ \\
$\mathrm{BIS}$ & $\mathrm{REMI}$ & $83 \pm 12$ & $77 \pm 11$ & $44 \pm 3^{*}$ & - \\
& $\mathrm{Control}$ & $94 \pm 5$ & $44 \pm 5^{*}$ & $44 \pm 2^{*}$ & - \\
& $\mathrm{N}_{2} \mathrm{O}$ & $93 \pm 6$ & $45 \pm 3^{*}$ & $44 \pm 3^{*}$ & - \\
\hline
\end{tabular}

Values are mean \pm SD. No statistical differences were observed in MAP, HR, and BIS at any time among the groups. $* \mathrm{P}<0.05$ versus before induction. 
in all three groups $(\mathrm{P}<0.05)$. Throughout the study, all stress hormone levels did not differ significantly at any time between groups.

\section{Discussion}

We determined the interaction of $\mathrm{N}_{2} \mathrm{O}$ or remifentanil with potent inhaled anesthetics on BIS guided anesthetic requirement and neuroendocrine response in SCI patients. The current study demonstrated that the adjunctive use of remifentanil or $\mathrm{N}_{2} \mathrm{O}$ similarly reduced sevoflurane requirements (by 31-39\%) before and during surgery, while BIS was kept between 40 and 50. The anesthetic requirement before surgery was similar to that during surgery when surgical stimulation was present, as all subjects were insensate below the level of injury where surgery was performed. It was also demonstrated that hemodynamic and stress hormone responses were comparable among groups, which rais-

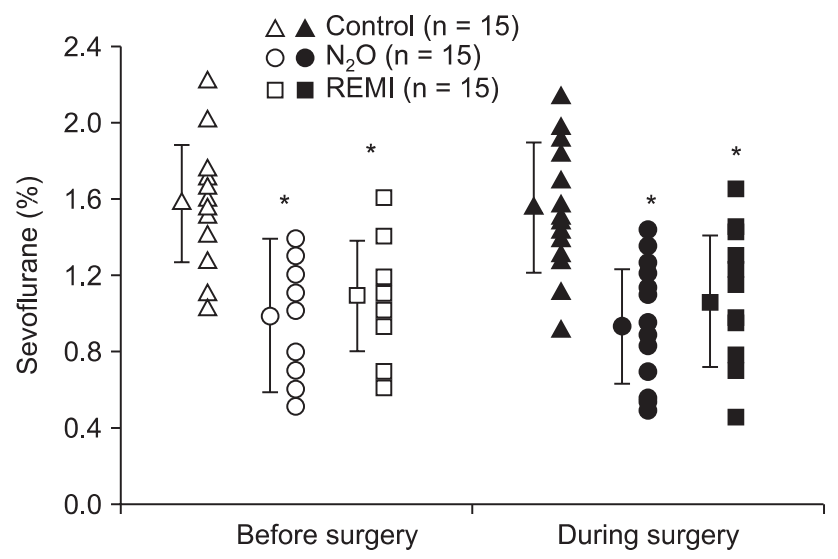

Fig. 3. Individual end-tidal sevoflurane concentration (\%) with group means and standard deviations before (open symbols) and during (closed symbols) surgery in patients given sevoflurane alone (control, triangles) or in combination with nitrous oxide $\left(\mathrm{N}_{2} \mathrm{O}\right.$, circles) or remifentanil (REMI, squares). $* \mathrm{P}<0.05$ versus control group. es doubts about the potential clinical benefits of antinociceptive agent remifentanil or $\mathrm{N}_{2} \mathrm{O}$ in SCI patients undergoing surgery below the level of injury. Major complications commonly seen during remifentanil infusions, such as intraoperative hypotension and bradycardia [8], were observed in only one patient.

It has been known that neither opioids [16] nor $\mathrm{N}_{2} \mathrm{O}$ [17], when given alone or in combination with inhaled anesthetics significantly affects the BIS values especially in the absence of noxious stimulation. However, clinically, $\mathrm{N}_{2} \mathrm{O}$ [18] and opioids [19] both have a hypnotic effect and this shifts the relationship between BIS values and responsiveness to stimulation to the right, that is, since BIS does not reflect their increased hypnotic effects, BIS values increase in $50 \%$ of the population that does not respond to a stimulus in the presence of $\mathrm{N}_{2} \mathrm{O}$ or opioids. In contrast, in the present study, $\mathrm{N}_{2} \mathrm{O}$ or remifentanil as an adjunct to sevoflurane significantly reduced the anesthetic requirements to achieve a determined level of hypnosis, as measured by BIS. This result is in agreement with that in a previous report, in which, despite the lack of effect of lidocaine on the BIS, IV lidocaine reduced the propofol requirements as guided by BIS, but only when surgical stimulus was present [19]. Likewise, although both adjuvants did not affect BIS by themselves, $\mathrm{N}_{2} \mathrm{O}$ was shown to decrease BIS when added to sevoflurane in healthy subjects undergoing surgery [20], and remifentanil was shown to prevent the increase in BIS associated with tracheal intubation under a constant regimen of propofol administration [21]. These findings indicate that $\mathrm{N}_{2} \mathrm{O}$ or remifentanil may affect BIS in the presence of noxious stimuli probably through an anti-nociceptive effect. All subjects were tracheally intubated in the present study, which may have introduced significant levels of noxious stimuli. It is likely that $\mathrm{N}_{2} \mathrm{O}$ or remifentanil may reduce sevoflurane requirements to a similar extent through antinociceptive interactions even in SCI patients.

Alternatively, the differential effects of $\mathrm{N}_{2} \mathrm{O}$ or remifentanil on BIS when combined with sevoflurane compared to those observed in healthy subjects $[16,17]$ could have resulted from

Table 3. Stress Hormone Concentrations before Induction of Anesthesia, before, During and 1 Hour after (Recovery) Surgery in Patients Given Sevoflurane Alone (Control, $\mathrm{n}=15)$ or in Combination with Nitrous Oxide $\left(\mathrm{N}_{2} \mathrm{O}, \mathrm{n}=15\right)$ or Remifentanil (REMI, $\mathrm{n}=15$ )

\begin{tabular}{|c|c|c|c|c|c|}
\hline Variable & Group & Before induction & Before surgery & During surgery & Recovery \\
\hline \multirow{3}{*}{ Norepinephrine (pg/ml) } & Control & $174 \pm 98$ & $176 \pm 97$ & $193 \pm 102$ & $191 \pm 139$ \\
\hline & $\mathrm{N}_{2} \mathrm{O}$ & $215 \pm 128$ & $200 \pm 108$ & $201 \pm 92$ & $235 \pm 104$ \\
\hline & REMI & $252 \pm 113$ & $223 \pm 99$ & $202 \pm 102$ & $295 \pm 180$ \\
\hline \multirow[t]{3}{*}{ Epinephrine (pg/ml) } & Control & $44 \pm 51$ & $22 \pm 21 *$ & $35 \pm 25$ & $35 \pm 26$ \\
\hline & $\mathrm{N}_{2} \mathrm{O}$ & $49 \pm 34$ & $21 \pm 16^{*}$ & $21 \pm 14^{*}$ & $57 \pm 29$ \\
\hline & REMI & $64 \pm 48$ & $30 \pm 31^{*}$ & $33 \pm 19$ & $46 \pm 27$ \\
\hline \multirow[t]{3}{*}{ Cortisol ( $\mu \mathrm{g} / \mathrm{dl})$} & Control & $15 \pm 5$ & $15 \pm 6$ & $10 \pm 5^{*}$ & $20 \pm 7^{*}$ \\
\hline & $\mathrm{N}_{2} \mathrm{O}$ & $13 \pm 4$ & $11 \pm 5$ & $9 \pm 3^{*}$ & $18 \pm 5^{*}$ \\
\hline & REMI & $17 \pm 5$ & $16 \pm 6$ & $10 \pm 3^{*}$ & $22 \pm 8^{*}$ \\
\hline
\end{tabular}

Values are mean $\pm \mathrm{SD} . * \mathrm{P}<0.05$ versus before induction. 
neuroplastic changes or reorganization within the cortex in SCI. Clinical and experimental evidences suggest that sensory deafferentation owing to SCI is associated with widespread changes in the somatosensory pathways and cortical information processing [4]. In this context, the extensive cortical reorganization may have altered the BIS (cortical) responses to $\mathrm{N}_{2} \mathrm{O}$ or remifentanil when combined with inhaled anesthetics, thereby possibly reducing anesthetic requirements by mechanisms to which BIS is sensitive.

In the current study, the amount of end-tidal sevoflurane needed to keep BIS between 40 and 50 was $1 \%$ in both the $\mathrm{N}_{2} \mathrm{O}$ and remifentanil groups. This anesthetic concentration is comparable with that in a previous report, which compared anesthetic requirements between SCI patients and uninjured control patients undergoing surgery below the level of injury and showed that anesthetic requirements were significantly reduced by $20-39 \%$ in SCI patients [10]. These results are in a good agreement with those in the study by Foffani et al. [22] who demonstrated a significant decrease in anesthetic requirements immediately after complete transection of the spinal cord in rats. Inhaled anesthetic is known to indirectly alter brain cortical activity induced by electrical stimulation of the reticular formation through its action in the spinal cord [12]. Taken together, it is suggested that an interruption of ascending transmission of nociceptive information by SCI increases susceptibility to anesthetics, thus possibly reducing their requirements for inducing unconsciousness and amnesia. In contrast, in patients with acute SCI undergoing surgery at the level of injury (i.e., twilight zone), anesthetic requirements were not different as compared with those in uninjured subjects [23]. Furthermore, the concentration of a volatile anesthetic needed to prevent autonomic dysreflexia was shown to be very high in high SCI patients (95\% effective sevoflurane concentration in $50 \% \mathrm{~N}_{2} \mathrm{O}$ of $3.83 \%$ ) [24]. Collectively, it is suggested that although SCI patients lose sensation below the level of injury, their anesthetic requirements are likely to depend on the site of surgery relative to the level of injury, kind of procedures or surgeries, or the time elapsed since injury.

The neuroendocrine response (i.e., stress response) to surgery that triggers hormonal secretion from the hypothalamus is activated by afferent signals (nociceptive pathways or humoral mediators) [25]. Its magnitude and duration depend on several factors such as severity and duration of surgical trauma, patients' age, anesthetic method, and surgical technique [26]. Since neural pathways that transmit afferent signals from the injured area and some sympathetic efferent fibers are interrupted in SCI patients, it was not unexpected to observe reduced catecholamine responses in the current study. Even after the recovery from anesthesia, catecholamine and hemodynamic responses were virtually abolished in all groups; and none of the patients experi- enced pain at the operative site, thereby confirming the result in the previous report [10].

Plasma cortisol concentrations increase rapidly after the beginning of surgery and reach the peak at emergence from anesthesia [27]. In contrast, an afferent neural blockade at spinal cord by using epidural anesthesia inhibits the release of cortisol in patients undergoing radical retropubic prostatectomy [28]. The present study also showed that the plasma cortisol concentrations were significantly decreased during surgery in each group, substantiating the previous notion that the hypothalamus-pituitary-adrenal axis is impaired in chronic SCI patients [29].

Nevertheless, plasma cortisol concentrations increased significantly above baseline values at 1 hour after the end of surgery in all groups. The discrepancy between catecholamine and cortisol responses indicates that the stress response may consist of discrete systems responding to different stimuli. Since the nociceptive neural pathway is already interrupted in SCI patients, some substances that are directly released in response to surgical trauma are likely to stimulate the release of cortisol. Indeed, surgically elicited increases in circulating cytokines were shown to contribute to perioperative increases in cortisol [30].

Remifentanil when used as an adjuvant to volatile anesthetics has some drawbacks such as intraoperative hypotension, bradycardia, and increased postoperative analgesic requirement due to acute opioid tolerance [8]. In the current study, hemodynamic and stress hormone responses were comparable between the two anesthetic adjuvant groups, and only one patient in the remifentanil group developed hypotension requiring treatment. Moreover, none of the patients in either group suffered from pain in the surgical wound during the recovery. An acute opioid tolerance is unlikely to be a clinical problem in spinal cordinjured patients. Therefore, remifentanil may be substituted for $\mathrm{N}_{2} \mathrm{O}$ during sevoflurane anesthesia even in spinal cord-injured patients. Nevertheless, since sevoflurane requirements were quite low, and hemodynamic and stress hormonal responses were similar between with and without two adjuvants in the present study; a different combination of anesthetics is unlikely to be superior to sevoflurane alone. However, the combination of anesthetic with either of the two adjuvants should be useful in situations in which the administration of high concentration of volatile anesthetic is required to cause severe hypotension (i.e., prevention of autonomic dysreflexia or surgery in the twilight zone).

The current study has a few limitations. First, SCI patients do not move even in the absence of adequate anesthesia and hemodynamic responses are altered due to the loss of supraspinal control of the sympathetic nervous system [3]. As such, an inhalation anesthetic requirement could not be quantified by somatic (e.g., MAC) or autonomic response (e.g., MAC needed 
to block the adrenergic response) in SCI. A BIS value less than 50 indicates an adequate depth of hypnosis to prevent recall for anesthetics including sevoflurane [15]. Therefore, the amount of sevoflurane needed to keep the BIS at 40-50 was used in the current study irrespective of the adequateness of anesthesia. Second, the lack of determination of anesthetic requirements in uninjured controls can be considered another important shortcoming of the study. However, we have previously demonstrated a $20-39 \%$ larger value in normal healthy controls than in SCI patients [10].

In summary, we showed that a TCI of $1.37 \mathrm{ng} / \mathrm{ml}$ remifentanil reduced anesthetic requirements to an extent similar to that achieved with $67 \% \mathrm{~N}_{2} \mathrm{O}$ without any clinically significant changes in hemodynamic and stress hormone responses in SCI patients undergoing surgery below the level of injury under sevoflurane anesthesia. This finding thus indicates that either remifentanil or $\mathrm{N}_{2} \mathrm{O}$ can be used as an adjunct to inhalation anesthetic in SCI patients.

\section{Acknowledgments}

This study was supported by a grant (\#CRI11026-1) from Chonnam National University Hospital Research Institute of Clinical Medicine, Jebong-ro 42, Gwangju 501-757, South Korea.

\section{References}

1. Pollock JE, Neal JM, Liu SS, Burkhead D, Polissar N. Sedation during spinal anesthesia. Anesthesiology 2000; 93: 728-34.

2. Hodgson PS, Liu SS, Gras TW. Does epidural anesthesia have general anesthetic effects? A prospective, randomized, double-blind, placebocontrolled trial. Anesthesiology 1999; 91: 1687-92.

3. Mathias CJ, Frankel HL. The cardiovascular system in tetraplegia and paraplegia. In: Spinal Cord Trauma, Hand Book of Clinical Neurology. 2nd ed. Edited by Frankel HL: New York, Elsevier Science Publishers. 1992, pp 435-56.

4. Aguilar J, Humanes-Valera D, Alonso-Calviño E, Yague, JG, Moxon KA, Oliviero A, et al. Spinal cord injury immediately changes the state of the brain. J Neurosci 2010; 30: 7528-37.

5. Hambly PR, Martin B. Anesthesia for chronic spinal cord lesions. Anaesthesia 1998; 53: 273-89.

6. Eger EI. Inhaled anesthetics: uptake and distribution. In: Miller's anesthesia. 7th ed. Edited by Miller RD: Philadelphia, Elsevier Churchill Livingstone. 2009, pp 539-59.

7. Kihara S, Yaguchi Y, Inomata S, Watanabe S, Brimacombe JR, Taguchi N, et al. Influence of nitrous oxide on minimum alveolar concentration of sevoflurane for laryngeal mask insertion in children. Anesthesiology 2003; 99: 1055-8.

8. Glass PS, Hardman D, Kamiyama Y, Quill TJ, Marton G, Donn KH, et al. Preliminary pharmacokinetics and pharmacodynamics of an ultra-short-acting opioid: remifentanil (GI87084B). Anesth Analg 1993; 77: 1031-40.

9. Lang E, Kapila A, Shlugman D, Hoke JF, Sebel PS, Glass PS. Reduction of isoflurane minimal alveolar concentration by remifentanil. Anesthesiology 1996; 85: 721-8.

10. Yoo K, Hwang J, Jeong S, Kim S, Bae H, Choi J, et al. Anesthetic requirements and stress hormone responses in spinal cord-injured patients undergoing surgery below the level of injury. Anesth Analg 2006; 102: 1223-8.

11. Park NG, Yoo KY, Jeong CW, Chung ST, Kim SJ, Kim WM, et al. Anesthetic requirements in chronic cord-injured patients undergoing surgery below the level of injury. Korean J Anesthesiol 2008; 54: S6-15.

12. Antognini JF, Atherley R, Carstens E. Isoflurane action in spinal cord indirectly depresses cortical activity associated with electrical stimulation of the reticular formation. Anesth Analg 2003; 96: 999-1003.

13. Antognini JF. The relationship among brain, spinal cord and anesthetic requirements. Med Hypotheses 1997; 48: 83-7.

14. Ohashi Y, Guo T, Orii R, Maze M, Fujinaga M. Brain stem opioidergic and GABAergic neurons mediate the antinociceptive effect of nitrous oxide in Fischer rats. Anesthesiology 2003; 99: 947-54.

15. Katoh T, Suzuki A, Ikeda K. Electroencephalograhic derivatives as a tool for predicting the depth of sedation and anesthesia induced by sevoflurane. Anesthesiology 1998; 88: 642-50.

16. Glass PS, Bloom M, Kearse L, Rosow C, Sebel P, Manberg P. Bispectral analysis measures sedation and memory effects of propofol, midazolam, isoflurane, and alfentanil in healthy volunteers. Anesthesiology 1997; 86: 836-47.

17. Barr G, Jakobsson JG, Owall A, Anderson RE. Nitrous oxide does not alter bispectral index: study with nitrous oxide as sole agent and as an adjunct to i.v. anaesthesia. Br J Anaesth 1999; 82: 827-30.

18. Karalapillai D, Leslie K, Umranikar A, Bjorksten AR. Nitrous oxide and anesthetic requirement for loss of response to command during propofol anesthesia. Anesth Analg 2006; 102: 1088-93.

19. Hans GA, Lauwick SM, Kaba A, Bonhomme V, Struys MM, Hans PC, et al. Intravenous lidocaine infusion reduces bispectral index-guided requirements of propofol only during surgical stimulation. Br J Anaesth 2010; 105: 471-9.

20. Ozcan MS, Ozcan MD, Khan QS, Thompson DM, Chetty PK. Does nitrous oxide affect bispectral index and state entropy when added to a propofol versus sevoflurane anesthetic? J Neurosurg Anesthesiol 2010; 22: 309-15. 
21. Guignard B, Menigaux C, Dupont X, Fletcher D, Chauvin M. The effect of remifentanil on the bispectral index change and hemodynamic responses after orotracheal intubation. Anesth Analg 2000; 90: 161-7.

22. Foffani G, Humanes-Valera D, Calderon-Muñoz F, Oliviero A, Aguilar J. Spinal cord injury immediately decreases anesthetic requirements in rats. Spinal Cord 2011; 49: 822-6.

23. Yoo KY, Kim TS, Jeong CW, Kim SJ, Jeong ST, Jeong SW, et al. Anaesthetic requirements and stress hormone responses in acute cordinjured patients undergoing surgery of the injured spine. Eur J Anaesthesiol 2009; 26: 304-10.

24. Yoo KY, Jeong CW, Kim SJ, Chung ST, Bae HB, Oh KJ, et al. Sevoflurane concentrations required to block autonomic hyperreflexia during transurethral litholapaxy in patients with complete spinal cord injury. Anesthesiology 2008; 108: 858-63.

25. Desborough JP, Hall GM. Endocrine response to surgery. In: Anesthesia Review. 10th ed. Edited by Kaufman L: Edinburgh, Churchill Livingstone. 1993, pp 131-48.

26. Shintani F, Kanba S, Nakaki T, Nibuya M, Kinoshita N, Suzuki E, et al. Interleukin-1 beta augments release of norepinephrine, dopamine and serotonin in rat anterior hypothalamus. J Neurosci 1993; 13: 3574-81.

27. Furuya K, Shimizu R, Hirabayashi Y, Ishii R, Fukuda H. Stress hormone responses to major intra-abdominal surgery during and immediately after sevoflurane-nitrous oxide anaesthesia in elderly patients. Can J Anaesth 1993; 40: 435-9.

28. Fant F, Tina E, Sandblom D, Andersson SO, Magnuson A, Hultgren-Hörnkvist E, et al. Thoracic epidural analgesia inhibits the neurohormonal but not the acute inflammatory stress response after radical retropubic prostatectomy. Br J Anaesth 2013; 110: 747-57.

29. Huang TS, Wang YH, Lee SH, Lai JS. Impaired hypothalamus-pituitary-adrenal axis in men with spinal cord injuries. Am J Phys Med Rehabil 1998; 77: 108-12.

30. Jones TH, Kennedy RL. Cytokines and hypothalamic-pituitary function. Cytokine 1993; 5: 531-8. 